\title{
Enquête
}

Archives de la revue Enquête

\section{2| 1985}

\section{Au fil de la lignée}

\section{Conclusion}

Au fil de la lignée, des identités ouvrières en question

\section{(2) OpenEdition}

\section{Journals}

Édition électronique

URL : http://journals.openedition.org/enquete/233

DOI : 10.4000/enquete.233

ISSN : 1953-809X

Éditeur :

Cercom, Éditions Parenthèses

Édition imprimée

Date de publication : 1 juin 1985

Référence électronique

"Conclusion », Enquête [En ligne], 2 | 1985, mis en ligne le 26 juin 2013, consulté le 20 avril 2019. URL : http://journals.openedition.org/enquete/233 ; DOI : 10.4000/enquete.233

Ce document a été généré automatiquement le 20 avril 2019. 


\title{
Conclusion
}

\author{
Au fil de la lignée, des identités ouvrières en question
}

1 Maintenant dites Fos et nous pensons, et vous pensez peut-être à ces familles. En 1982 et 1983, lorsque nous les avons rencontrées, elles étaient à la croisée de destinées bien différentes.

2 Si quelques-uns encore (les Nouge, les Illico) tentent de s'accrocher à des systèmes d'identité bien stables, tous les autres nous signifient, à travers leurs rêves les plus fous comme à travers leurs projets les mieux construits en apparence, leur désir de ne pas être assignés à identité socio-professionnelle et leur refus de l'enfermement pratique ou symbolique dans le monde de l'usine. Que ces rêves et projets les plus divers (le principal et de loin étant quand même celui de «la mise à son compte » avec l'ouverture d'un petit commerce ou d'un atelier) soient inscrits dans le roman familial et facilités par la faible hérédité industrielle ou ouvrière des familles que nous avons rencontrées semble faire peu de doute. Mais l'essentiel n'est peut-être pas là.

3 Il réside dans le fait que les rapports des individus à leur condition sociale sont beaucoup plus contradictoires qu'on ne le pense souvent. Regardez Robert Rainard: sa conscience d'appartenir à une classe semble totale, son référent identitaire majeur est bien le mouvement ouvrier dans ses formes les plus classiques, et pourtant ne pense-t-il pas lui aussi à « se mettre à son compte »?

4 S'agirait-il d'un phénomène ancien que l'on ne fait que redécouvrir? S'agirait-il d'une réaction à une situation où la perte d'efficacité des modes traditionnels de résistance ouvrière et l'accumulation d'expériences d'échecs de l'action collective conduisent à la recherche de solutions familiales? Ces rapports contradictoires vécus par les individus d'une génération qui connaît au mi-temps de sa vie les restructurations que l'on a évoquées ne seraient-ils que le reflet de la recomposition du champ des positions sociales dans l'entreprise et l'expression de l'incertitude créée par de nouvelles règles du jeu qui mettent en avant la remise en cause des situations acquises, la mobilité, la polyvalence, l'acquisition permanente de nouveaux savoirs en cours de vie professionnelle?

Phénomènes sociétaux certes, mais combien accusés chez ces familles de travailleurs de la SOLMER ; cette usine de pointe, née dans l'euphorie du début des années soixante-dix, avait à peine donné vie aux grands espoirs de promotion à l'intérieur du monde ouvrier - 
promotion bien souvent largement amorcée au cours des deux précédentes décennies que la crise économique, particulièrement forte dans la sidérurgie, resserre brutalement le champ des possibles et redéfinit toutes les règles du jeu.

6 Le rôle croissant de la socialisation scolaire, l'évolution rapide des qualifications et la réactualisation permanente des savoirs au cours de la vie n'autorisent plus les mêmes cristallisations des identités professionnelles. Pour une génération qui connait à la fois les ruptures dans le cours des lignées et une instabilité des points de repère professionnels, pères et pairs ne constituent plus les mêmes figures d'identification et des motifs de mobilisation. Phénomènes de génération passagers ou nouvelle donne structurelle? la question reste ouverte.

7 Au moment où nous quittons ces lieux, la fascination du mastodonte industriel a disparu, les images de Métropolis s'évanouissent. Apparaissent maintenant celles de ces personnages (pour lesquels toute ressemblance avec des personnes vivantes est absolument garantie) qui tournent en ce moment même, à leur insu peut-être, une page de l'histoire du monde du travail industriel.

Dans leurs combats quotidiens, leurs aspirations, ils nous ont appris combien il est vain de vouloir continuer à tenir des discours édifiants sur les besoins de la classe ouvrière, sur l'identité ouvrière, sur la culture ouvrière.

9 Laisser sa vie ouverte à de nouveaux possibles ; s'échapper des pesantes routines ; ne pas être assigné à identité ; ne pas voir la moindre de ses pensées, le moindre de ses actes réduits par avance à la réalisation d'un destin sociologique; voilà des exigences identitaires profondes que bien des analystes des «besoins» "ouvriers" risquent d'oublier en chemin. 